\title{
OPERATORS ON ALMOST HERMITIAN MANIFOLDS
}

\author{
YÔSUKE OGAWA
}

\section{Introduction}

Recently C. C. Hsiung [1] showed, among other results on the realization of the complex Laplace-Beltrami operator $\square$ on an almost Hermitian space, that if for an almost Hermitian structure the relation $\square=\Delta / 2$ holds for all forms of degrees 0 and 1 , then the structure is Kaehlerian, where $\Delta$ denotes the real Laplace-Beltrami operator; this result was a conjecture for some time and is an improvement of a theorem by Kodaira-Spencer [2]. In the present paper, we point out that the two definitions of the operator $\square$ given by Kodaira-Spencer and C. C. Hsiung respectively are different, and extend the above result of Hsiung by showing that for an almost Hermitian structure if $\square$ according to either definition is real on all forms of degrees 0 and 1 , then the structure is Kaehlerian ${ }^{1}$.

Let $\prod_{r, s}$ be the projection mapping onto the subspace composed of elements of type $(r, s)$ (see $\S 1)$, and $\partial$ the skew-derivation of dgree 1 , which coincides on functions with $\prod_{1,0} d$ and satisfies the relation $\partial d+d \partial=0$. Then the definition of the integrable condition of the almost complex structure is given by $\partial^{2}=0$, [2]. By investigating the real and imaginary parts of the operator $\partial$, we express an equivalent condition of $\partial^{2}=0$ in terms of some real operators, and give a condition on a real operator for an almost Hermitian structure to be Kaehlerian.

\section{Definitions}

Let $M^{n}$ be a Riemannian space, denote its fundamental metric tensor by $g_{\lambda \mu}$, and put $g=\operatorname{det}\left|g_{\lambda \mu}\right|$. (In the following the Greek indices $\lambda, \mu, \nu, \cdots$ run from 1 to $n$, the dimension of the space.) Let $\varepsilon_{\lambda_{1} \ldots \lambda_{p}}^{\mu_{1} \ldots \mu_{p}}$ be the generalized Kronecker's delta, $\varepsilon_{\lambda_{1} \cdots \lambda_{n}}$ stand for $\varepsilon_{\lambda_{1} \cdots \lambda_{n}}^{1 \cdots \cdots}$, and $\mathscr{F}^{p}$ be the algebra of differential $p$-forms on $M^{n}$. Then the exterior differentiation $d: \mathscr{F}^{p} \rightarrow \mathscr{F}^{p+1}$ and the adjoint

\footnotetext{
Received November 8, 1968.

1 After this paper was written, Hsiung informed me that at the Summer Institute on Relativity and Differential Geometry sponsored by the American Mathematical Society and the National Science Foundation at the University of California at Santa Barbara in 1962 he had raised the question: If $\square$ for an almost Hermitian structure is real, is the structure Kaehlerian?
} 
operator $*: \mathscr{F}^{p} \rightarrow \mathscr{F}^{n-p}$ can be written for a $p$-form $u=\left(u_{\lambda_{1} \cdots \lambda_{p}}\right)$ by

$$
\begin{aligned}
& (d u)_{\lambda_{0} \cdots \lambda_{p}}=(1 / p !) \varepsilon_{\lambda_{0} \ldots{ }_{2} p}^{\rho \mu_{1} \cdots{ }_{\rho}} \nabla_{\rho} u_{\mu_{1} \ldots \mu_{p}}, \\
& (* u)_{\lambda_{1} \ldots \lambda_{n-p}}=(1 / p !) \sqrt{g} g^{\rho_{1} \mu_{1}} \ldots g^{\rho p^{\mu} p} u_{\rho_{1} \ldots \rho_{p}} \varepsilon_{\mu_{1} \ldots \mu_{p \lambda_{1} \ldots \lambda_{n-p}}},
\end{aligned}
$$

where $\nabla_{2}$ denotes the covariant derivation with respect to the Riemann connection. The exterior co-differentiation $\delta: \mathscr{F}^{p} \rightarrow \mathscr{F}^{p-1}$ defined by

$$
\delta=(-1)^{n p+n+1} * d *
$$

can be expressed locally as

$$
(\delta u)_{\lambda_{2} \cdots \lambda_{p}}=-\nabla^{\rho} u_{\rho \lambda_{2} \cdots \lambda_{p}} .
$$

Let $\Delta$ be the Laplace-Beltrami operator defined by

$$
\Delta=d \delta+\delta d,
$$

then by means of (1.1) and (1.3) it is easy to verify that for a form $u$ of degree $p$

$$
(\Delta u)_{\lambda_{1} \cdots \lambda_{p}}=-\nabla^{\rho} \nabla_{\rho} u_{\lambda_{1} \cdots \lambda_{p}}+\sum_{i=1}^{p} R_{\lambda_{i}}{ }^{\rho} u_{\lambda_{1} \cdots \hat{\rho} \cdots \lambda_{p}} \stackrel{i}{i}+\sum_{i<j} R_{\lambda_{i} \lambda_{j}{ }^{\rho \sigma}} u_{\lambda_{1} \cdots \hat{\rho} \cdots \hat{\rho} \cdots \lambda_{p}} \stackrel{i}{j}
$$

holds, where $R_{\lambda \mu \nu \omega}$ (or $R_{\lambda \mu}$ ) is the curvature (or Ricci) tensor of the Riemann connection, $u_{\lambda_{1} \ldots \hat{\rho} \cdots \lambda_{p}} \stackrel{i}{i}$ indicates that the subscript $\rho$ replaces the subscript $\lambda_{i}$, and $u_{\lambda_{1} \ldots \hat{\alpha} \cdots \lambda_{p}}$ indicates that the subscript $\lambda_{\alpha}$ is deleted.

If a Riemannian space $M^{n}$ admits an almost complex structure $\varphi_{\lambda}{ }^{\mu}$ satisfying

$$
g_{\rho \sigma} \varphi_{\lambda}{ }^{\rho} \varphi_{\mu}{ }^{\sigma}=g_{\lambda \mu},
$$

then it is called an almost Hermitian space. Let $T^{c}(M)$ be a complexification of the tangent space of $M^{n}$, and denote by $\mathscr{F}_{c}{ }^{p}$ the (complexified) differential $p$-forms, that is, the complex-valued functions defined on $T^{c}(M) \wedge \ldots$ $\wedge T^{c}(M)$. For non-negative integers $r, s$ we define the projection mapping $\Pi: \mathscr{F}_{c}{ }^{p} \rightarrow \mathscr{F}_{c}{ }^{p}$ where $p=r+s$ as follows. At first

$$
\prod_{1,0}{ }^{\mu}=(1 / 2)\left(\delta_{\lambda}{ }^{\mu}-\sqrt{-1} \varphi_{\lambda}{ }^{\mu}\right)
$$

and its conjugate

$$
\prod_{0,1}{ }^{\mu}=\bar{\Pi}_{1,0}{ }^{\mu}=(1 / 2)\left(\delta_{\lambda}{ }^{\mu}+\sqrt{-1} \varphi_{\lambda}{ }^{\mu}\right)
$$

which will be abbreviated to $\Pi$ and $\bar{\Pi}$ respectively. Then for a $p$-form $u$ of 
$\mathscr{F}_{c}^{p}$, we define

$$
\begin{aligned}
& \left(\prod_{r, s} u\right)_{\lambda_{1} \cdots \lambda_{p}}=(1 / p !) \prod_{r, s} \prod_{\lambda_{1} \cdots \lambda_{p}}^{\mu_{1} \cdots{ }^{\mu} p} u_{\mu_{1} \cdots \mu_{p}}
\end{aligned}
$$

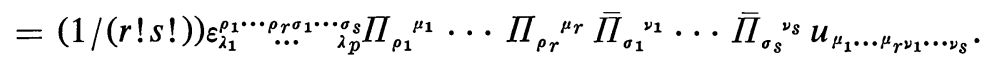

A $p$-form $u$ of $\mathscr{F}_{c}{ }^{p}$ is called of type $(r, s)$ if it satisfies $\Pi_{r, s} u=u$.

Lemma 1.1. In an almost complex space, for any set of functions $u_{\lambda_{1} \cdots \lambda_{p}}$ we have

$$
\sum_{k=0}^{p}\left(\prod_{p-k, k} u\right)_{\lambda_{1} \cdots \lambda_{p}}=u_{\lambda_{1} \cdots \lambda_{p}} .
$$

Proof. We prove by induction. Suppose that (1.10) is true for all sets of functions $u_{\lambda_{1} \cdots \lambda_{r}}$ for $r \leq p$; for convenience those functions are said to be of degree $r \leq p$. Now let $u=\left(u_{\lambda_{0} \ldots \lambda_{p}}\right)$ be a set of functions of degree $p+1$. Then we have

$$
\begin{aligned}
& (1 /(p+1) !) \sum_{k=0}^{p+1} \prod_{p+1-k, k} \underset{\lambda_{0} \cdots \lambda_{p}}{\mu_{0} \cdots \mu} p u_{\mu_{0} \cdots \mu} \\
& =(1 /(p+1) !) \sum_{k=0}^{p+1}{ }_{p+1} C_{k} \varepsilon_{\lambda_{0}}^{\rho_{1} \cdots \rho_{p+1} \ldots k \sigma_{1} \cdots{ }_{\lambda p} k} \Pi_{\rho_{1}}^{\mu_{1}} \cdots \Pi_{\rho_{p+1-k}}{ }^{\mu_{p+1-k}}
\end{aligned}
$$

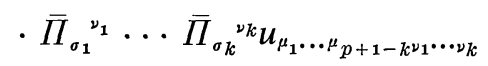

$$
\begin{aligned}
& =(1 /(p+1) !)\left[\varepsilon_{\lambda_{0} \cdots \lambda_{p}}^{\rho_{0} \ldots \rho} \Pi_{\rho_{0}}{ }^{\mu_{0}} \cdots \Pi_{\rho_{p}}{ }^{\mu} p u_{\mu_{0} \cdots \mu_{p}}+\varepsilon_{\lambda_{0} \cdots \lambda_{p}}^{\sigma_{0} \cdots{ }_{p} p} \bar{\Pi}_{\sigma_{0}}{ }^{\nu_{0}} \cdots \bar{\Pi}_{\sigma_{p}}{ }^{{ }^{\nu} p} u_{\nu_{0} \cdots \nu p}\right. \\
& +\sum_{k=1}^{p}\left({ }_{p} C_{k+p} C_{k-1}\right) \varepsilon_{\lambda_{0}}^{\rho_{1} \cdots \rho_{p+1-k} \sigma_{1} \cdots{ }_{\lambda p} k} \Pi_{\rho_{1}}^{\mu_{1}} \cdots \Pi_{\rho_{p+1-k}{ }^{\mu} p+1-k} \bar{\Pi}_{\sigma_{1}}^{{ }^{\nu_{1}}} \\
& \text {. . . } \left.\bar{\Pi}_{\sigma_{k}}^{{ }^{\nu} k} u_{\mu_{1} \ldots \mu_{p+1-k^{\nu}} \cdots \nu k}\right] \text {, }
\end{aligned}
$$

where ${ }_{p+1} C_{k}$ denotes the number of combinations of $p+1$ different things taken $p$ at a time. Considering the assumption of the induction, we get

$$
\begin{aligned}
& \varepsilon_{\lambda_{0} \cdots \lambda_{p}}^{\rho_{0} \ldots \rho_{p}} \Pi_{\rho_{0}}^{\mu_{0}} \cdots \Pi_{\rho_{p}}{ }^{\mu} p u_{\mu_{0} \ldots \mu_{p}}
\end{aligned}
$$

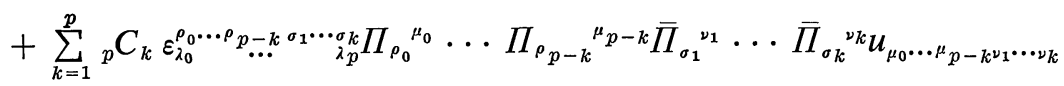

$$
\begin{aligned}
& =\sum_{\alpha=0}^{p}(-1)^{\alpha} p ! \Pi_{\lambda_{\alpha}}{ }^{\rho} u_{\rho \lambda_{0} \cdots \hat{\alpha} \cdots \lambda_{p}} \text {. }
\end{aligned}
$$

In a similar way, we have

$$
\begin{aligned}
& \varepsilon_{\lambda_{0} \cdots \lambda_{p}}^{\sigma_{0} \cdots p} \bar{\Pi}_{\sigma_{0}}^{\nu_{0}} \cdots \bar{\Pi}_{\sigma_{p}}^{\nu p} u_{\nu_{0} \cdots \nu p}
\end{aligned}
$$

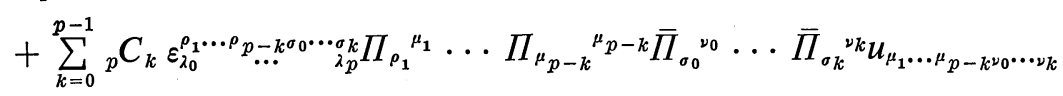

$$
\begin{aligned}
& =\sum_{\alpha=0}^{p}(-1)^{\alpha} p ! \bar{\Pi}_{\lambda_{\alpha}{ }^{\sigma}} u_{\sigma \lambda_{0} \cdots \hat{\alpha} \cdots \lambda_{p}} .
\end{aligned}
$$


Hence we have

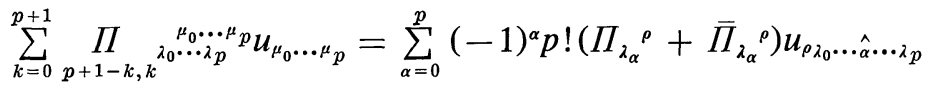

$$
\begin{aligned}
& =(p+1) ! u_{\lambda_{0} \cdots \lambda_{p}},
\end{aligned}
$$

which shows that (1.10) is true for any set of functions $u_{\lambda_{0} \ldots \lambda_{p}}$ of degree $p+1$.

From the proof of the above lemma, we see that in an almost complex space

$$
\sum_{k=0}^{p}{ }_{p} C_{k} \varepsilon_{\lambda_{1} \cdots \lambda_{p}}^{\rho_{1} \ldots \rho} \Pi_{\rho_{1}}^{{ }^{\mu}} \cdots \Pi_{\rho_{k}}{ }^{\mu_{k}} \bar{\Pi}_{\rho_{k+1}}{ }^{\mu_{k+1}} \cdots \bar{\Pi}_{\rho_{p}}{ }^{\mu} p u_{\mu_{1} \cdots \mu_{p}}=\varepsilon_{\lambda_{1} \cdots \lambda_{p} p}^{\mu_{1} \cdots{ }^{\mu} p} u_{\mu_{1} \ldots \mu_{p}}
$$

holds for any $p$-form $u_{\mu_{1} \cdots \mu_{p}}, 1 \leq p \leq n$.

Now we define the operators $d_{1}: \mathscr{F}_{c}{ }^{p} \rightarrow \mathscr{F}_{c}{ }^{p+1}$ of type $(1,0)$ and $d_{2}: \mathscr{F}_{c}{ }^{p}$ $\rightarrow \mathscr{F}_{c}{ }^{p+1}$ of type $(2,-1)$ in accordance with [2] by

$$
\begin{aligned}
& d_{1}=\sum_{r+s=p} \prod_{r+1, s} d \prod_{r, s}, \\
& d_{2}=\sum_{r+s=p} \prod_{r+2, s-1} d \prod_{r, s} .
\end{aligned}
$$

We denote the conjugate operator of $d_{1}$ (or $d_{2}$ ) by $\bar{d}_{1}$ (or $\bar{d}_{2}$ ).

Lemma 1.2. In an almost complex space, on $\mathscr{F}_{c}{ }^{p}$ we have

$$
\prod_{r+3, s-2} d \prod_{r, s}=0
$$

where $r+s=p$.

Proof. For any $p$-form $u$, we have

$$
\begin{aligned}
& \left(\prod_{r+3, s-1} d \prod_{r, s} u\right)_{\lambda_{0} \cdots \lambda_{p}}
\end{aligned}
$$

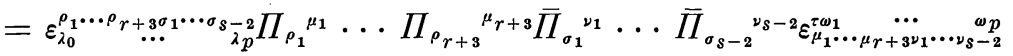

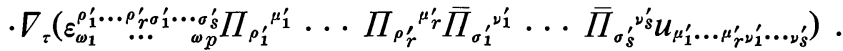

On the right hand side, there appear such terms of the types as

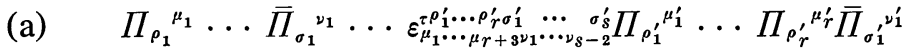

$$
\cdots \bar{\Pi}_{\sigma_{s}{ }^{\prime} s} \nabla_{\tau} u_{\mu_{1}^{\prime} \ldots \mu_{r \nu^{\prime}}^{\prime} \cdots \nu_{s}^{\prime}}
$$

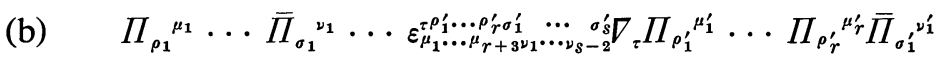

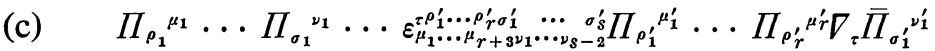

$$
\cdots \bar{\Pi}_{\sigma_{s}^{\prime}}^{\nu^{\prime}} \boldsymbol{u}_{\mu_{1}^{\prime} \ldots \mu_{r}^{\prime} \nu_{1}^{\prime} \ldots \nu_{s}^{\prime}} \text {. }
$$

Thus in (a), at least one of terms of the index pairs $\left(\sigma_{1}^{\prime}, \cdots, \sigma_{s}^{\prime}\right)$ must coincides 
with that of $\left(\mu_{1}, \cdots, \mu_{r+3}\right)$, and from the fact that

$$
\Pi_{\rho}^{\mu} \bar{\Pi}_{\mu}^{{ }^{\prime \prime}}=0
$$

we can conclude that all terms in (a) are zero. For the same reason all terms in (b) and (c) are also zero. Hence the lemma is proved.

Taking account of Lemmas 1.1 and 1.2., we see that

$$
d=d_{1}+d_{2}+\bar{d}_{1}+\bar{d}_{2}
$$

holds [2].

Next the definitions of complex analogues of the real operators $d$ and $\delta$ in the sense of Kodaira-Spencer [2] are as follows:

$$
\begin{aligned}
& \partial=2 d_{2}+d_{1}-\bar{d}_{2}, \\
& D=-* \partial * .
\end{aligned}
$$

On the other hand, C. C. Hsiung defined them by the following operators

$$
\begin{aligned}
& (\partial u)_{\lambda_{0} \cdots \lambda_{p}}=(1 / p !) \sum_{r+s=p} \prod_{r+1, s} \underset{\lambda_{0} \cdots \mu_{p} \cdots \mu_{p}}{\rho \mu_{1} \cdots \lambda^{\prime}} \Pi_{\rho}{ }^{\sigma} \nabla_{\sigma} u_{\mu_{1} \ldots \mu_{p}}, \\
& (\mathfrak{D} u)_{\lambda_{2} \cdots \lambda_{p}}=-\sum_{r+s=p} \prod_{r, s} \prod_{\rho_{\lambda_{2} \cdots \lambda_{p}} \mu_{1} \cdots \mu_{p}} \bar{\Pi}_{\sigma}^{\rho} \nabla^{\sigma} u_{\mu_{1} \cdots \mu_{p}}
\end{aligned}
$$

for a $p$-form $u=\left(u_{\lambda_{1} \cdots \lambda_{p}}\right)$. Later we shall show that the relation

$$
\mathfrak{D}=-* \partial *
$$

is valid.

In order to make clear the real and imaginary parts of the operators $\partial$ and $\partial$ and the others, we define the following more operators in an almost Hermitian space $M^{n}$ for a $p$-form $u=\left(u_{\lambda_{1} \ldots \lambda_{p}}\right)$ :

$$
\begin{aligned}
& (\Gamma u)_{\lambda_{0} \cdots \lambda_{p}}=\sum_{\alpha=0}^{p}(-1)^{\alpha} \varphi_{\lambda_{\alpha}}{ }^{\rho} \nabla_{\rho} u_{\lambda_{0} \ldots \hat{\alpha} \cdots \lambda_{p}} \quad(p \geq 0) \\
& (\gamma u)_{\lambda_{0} \cdots \lambda_{p}}=\sum_{\alpha \neq \beta}(-1)^{\alpha} \nabla_{\lambda_{\alpha}} \varphi_{\lambda_{\beta}}{ }^{\rho} u_{\lambda_{0} \ldots \hat{\alpha} \cdots \hat{\rho}^{\prime} \cdots \lambda_{p}} \quad(p \geq 1)
\end{aligned}
$$

from $\mathscr{F}^{p}$ to $\mathscr{F}^{p+1}$;

$$
\begin{array}{ll}
(D u)_{\lambda_{2} \cdots \lambda_{p} p}=\varphi^{\rho \sigma} \nabla_{\rho} u_{\sigma \lambda_{2} \cdots \lambda_{p} p} & (p \geq 1), \\
(\vartheta u)_{\lambda_{2} \cdots \lambda_{p}}=\sum_{\alpha=2}^{p}(-1)^{\alpha} \nabla^{\rho} \varphi_{\lambda_{\alpha}}^{\sigma} u_{\rho \sigma \lambda_{2} \cdots \hat{\alpha} \cdots \lambda_{p} p} & (p \geq 2)
\end{array}
$$

from $\mathscr{F}^{p}$ to $\mathscr{F}^{p-1}$ with 


$$
\begin{aligned}
\gamma f & =D f=\vartheta f=0, & & f \in \mathscr{F}^{0}, \\
\vartheta u & =0, & & u \in \mathscr{F}^{1}
\end{aligned}
$$

for forms of lower degrees; finally

$$
\begin{aligned}
& (\Phi u)_{\lambda_{1} \cdots \lambda_{p}}=\sum_{\alpha=1}^{p} \varphi_{\lambda_{\alpha}}{ }^{\rho} u_{\lambda_{1} \ldots \hat{\rho} \cdots \lambda_{p}} \stackrel{\alpha}{\alpha} \quad(p \geq 1), \\
& (\Psi u)_{\lambda_{1} \cdots \lambda_{p}}=\varphi_{\lambda_{1}}^{{ }^{\rho_{1}}} \cdots \varphi_{\lambda_{p}}{ }^{{ }{ }^{p}} \boldsymbol{u}_{\rho_{1} \ldots \rho_{p}} \quad(p \geq 1)
\end{aligned}
$$

from $\mathscr{F}^{p}$ to $\mathscr{F}^{p}$.

\section{Lemmas}

We study the properties of the operators defined in $\S 1$.

Lemma 2.1. In an almost Hermitian space, the operator $\Gamma$ is a skewderivation and satisfies

$$
* \Gamma *=-D
$$

Proof. The same calculation as in the proof of Lemma 2.2 in [4] shows that $\Gamma$ is a skew-derivation, and that, for any $p$-form $u=\left(u_{\lambda_{1} \cdots \lambda_{p}}\right)$,

$$
(* \Gamma * u)_{\lambda_{2} \cdots \lambda_{p}}=(-1)^{n p+n+1}(D u)_{\lambda_{2} \cdots \lambda_{p}}
$$

holds, where $n$ is the dimension of the space. Since $n$ is even, we have (2.1) immediately.

Lemma 2.2. In an almost Hermitian space, the operator $\Phi$ is a derivation and satisfies, for any p-form $u_{p}$,

$$
\begin{aligned}
* \Phi * u_{p} & =(-1)^{p} \Phi u_{p}, \\
d \Phi-\Phi d & =-\Gamma+\gamma .
\end{aligned}
$$

Proof. By a directive calculation with respect to an orthonormal local coordinate system for any $p$-form $u=\left(u_{\lambda_{1} \ldots \lambda_{p}}\right)$ we have

$$
\begin{aligned}
& (* \Phi * u)_{\lambda_{1} \cdots \lambda_{p}}=(1 /(n-p) ! p !) g g^{\mu_{1} \rho_{1}} \cdots g^{\mu n-p^{\rho} n-p} g^{\nu_{1} \tau_{1}} \cdots g^{\nu p^{\tau} p} u_{\nu_{1} \ldots \nu} \\
& \cdot \sum_{i=1}^{n-p} \varphi_{\mu_{i}{ }^{\sigma}} \varepsilon_{\tau_{1} \ldots \tau_{p}} p^{\mu_{1} \ldots \hat{\sigma} \cdots \mu_{n-p}}{ }^{i} \varepsilon_{\rho_{1} \ldots \rho_{n-p \lambda_{1}} \cdots \lambda_{p}}
\end{aligned}
$$

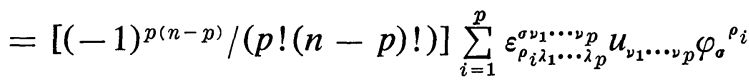

$$
\begin{aligned}
& =\left((-1)^{p(n-p)} / p !\right) \sum_{i=1}^{p} \varepsilon_{\lambda_{1} \ldots \hat{\rho}_{1} \ldots \lambda_{p}}^{\nu_{1} \ldots{ }^{\nu} p} \varphi_{\lambda_{i}}{ }^{\rho} u_{\nu_{1} \ldots \nu_{p}} \\
& =(-1)^{p(n-p)}(\Phi u)_{\lambda_{1} \cdots \lambda_{p}} \text {. }
\end{aligned}
$$


Since $n$ is even, we have $(-1)^{p(n-p)}=(-1)^{p}$, and thus $(2.2)$ is proved.

As for (3.3), we get

$$
\begin{aligned}
& (d \Phi u)_{\lambda_{0} \cdots \lambda_{p}}=\nabla_{\lambda_{0}} \varphi_{\lambda_{i}}{ }^{\rho} u_{\lambda_{1} \ldots \hat{\rho} \cdots \lambda_{p}} \stackrel{i}{i}-\nabla_{\lambda_{i}} \varphi_{\lambda_{0}}{ }^{\rho} u_{\lambda_{1} \cdots \hat{\rho} \cdots \lambda_{p}} \stackrel{i}{i}-\sum_{i \neq j} \nabla_{\lambda_{i}} \varphi_{\lambda_{j}}{ }^{\rho} u_{\lambda_{1} \cdots \hat{\lambda}_{0} \cdots \hat{\rho} \cdots \lambda_{p}} \stackrel{i}{j}
\end{aligned}
$$

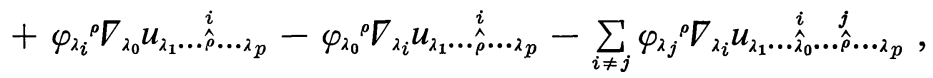

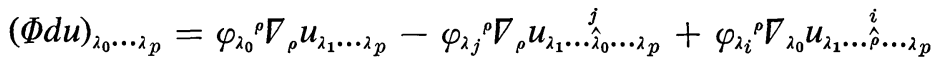

$$
\begin{aligned}
& -\varphi_{\lambda_{0}}{ }^{\rho} \nabla_{\lambda_{i}} u_{\lambda_{1} \ldots \hat{\rho} \ldots \lambda_{p}}^{i}-\sum_{i \neq j} \varphi_{\lambda_{j}{ }^{\rho}} \nabla_{\lambda_{i}} u_{\lambda_{0} \ldots \hat{\rho} \ldots \hat{\beta}_{0} \cdots \lambda_{p}} \stackrel{i}{i}
\end{aligned}
$$

Hence it follows that

$$
\begin{aligned}
(d \Phi u-\Phi d u)_{\lambda_{0} \cdots \lambda_{p}}= & \left(\nabla_{\lambda_{0}} \varphi_{\lambda_{i}}{ }^{\rho}-\nabla_{\lambda_{i}} \varphi_{\lambda_{0}}{ }^{\rho}\right) u_{\lambda_{1} \cdots \hat{\rho} \cdots \lambda_{p}}-\sum_{\alpha}(-1)^{\alpha} \varphi_{\lambda_{\alpha}}{ }^{\rho} \nabla_{\rho} u_{\lambda_{0} \cdots \hat{\alpha} \cdots \lambda_{p}} \\
& +\sum_{i<j}(-1)^{i}\left(\nabla_{\lambda_{i}} \varphi_{\lambda_{j}}{ }^{\rho}-\nabla_{\lambda_{j}} \varphi_{\lambda_{i}}{ }^{\rho}\right) u_{\lambda_{0} \lambda_{1} \cdots \hat{i} \cdots \hat{\rho}^{j} \cdots \lambda_{p}} \\
= & \sum_{\alpha<\beta}(-1)^{\alpha}\left(\nabla_{\lambda_{\alpha}} \varphi_{\lambda_{\beta}}{ }^{\rho}-\nabla_{\lambda_{\beta}} \varphi_{\lambda_{\alpha}}{ }^{\rho}\right) u_{\lambda_{0} \cdots \hat{\alpha} \cdots \hat{\rho} \cdots \lambda_{p}}-\sum_{\alpha}(-1)^{\alpha} \varphi_{\lambda_{\alpha}}{ }^{\rho} \nabla_{\rho} u_{\lambda_{0} \cdots \hat{\alpha} \cdots \lambda_{p}} \\
= & (\gamma u-\Gamma u)_{\lambda_{0} \cdots \lambda_{p}} .
\end{aligned}
$$

Next considering the following relation

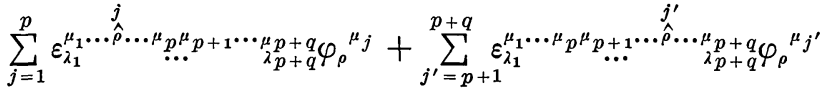

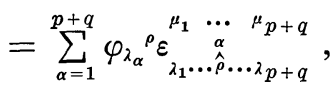

we have

$$
\begin{aligned}
& (\Phi u \wedge v)_{\lambda_{1} \cdots \lambda_{p+q}}+(u \wedge \Phi v)_{\lambda_{1} \cdots \lambda_{p+q}}
\end{aligned}
$$

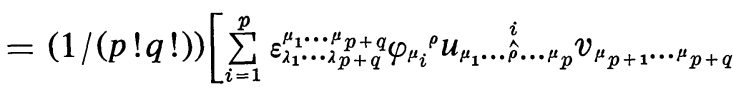

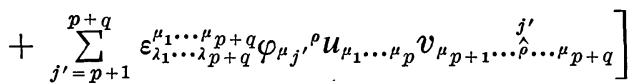

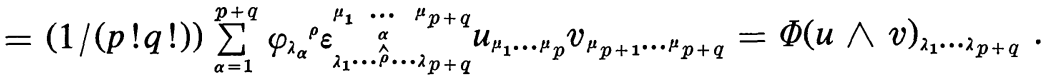

Thus the operator $\Phi$ is a derivation.

Corollary 2.3. In an almost Hermitian space, the operator $\gamma$ is a skewderivation.

Proof. Since $\Phi$ is a derivation, $\Phi d-d \Phi$ is a skew-derivation. By virtue of Lemma 2.1 and equation (2.3), $\gamma$ is a skew-derivation. 
Corollary 2.4. In an almost Hermitian space, the relation

$$
d \Gamma+\Gamma d=d \gamma+\gamma d
$$

holds.

Proof. (2.3) implies

$$
-d \Phi d=d(-\Gamma+\gamma), \quad d \Phi d=(-\Gamma+\gamma) d,
$$

from which follows (2.4) immediately.

Lemma 2.5. In an almost Hermitian space, we have

$$
* \gamma *=-\vartheta-i(\delta \varphi)
$$

where $i(\delta \varphi)$ denotes the inner product with respect to a 1 -form $\delta \varphi\left(\varphi=\varphi_{\lambda \mu}\right)$.

Proof. From the definition of $\gamma$, we have, for a $p$-form $u$,

$$
(\gamma u)_{\lambda_{0} \ldots \lambda_{p}}=\sum_{\alpha<\beta}(-1)^{\alpha} A_{\lambda_{\alpha} \lambda_{\beta}}{ }^{\rho} u_{\lambda_{0} \ldots \hat{\alpha} \ldots \hat{\rho}_{\ldots \lambda} \ldots \lambda_{p}},
$$

where we put $A_{\lambda \mu}{ }^{\rho}=\nabla_{\lambda} \varphi_{\mu}{ }^{\rho}-\nabla_{\mu} \varphi_{\lambda}{ }^{\rho}$. Therefore we have

$$
\begin{aligned}
& (* \gamma * u)_{\lambda_{2} \cdots \lambda_{p}} \\
& =[g /((n-p+1) ! p !)] \sum_{1 \leq i<j \leq n-p+1}(-1)^{i-1} A_{\mu_{i}{ }^{\mu} j}{ }^{\sigma} g^{\rho_{1} \mu_{1}} \cdots g^{\rho_{n-p+1} \mu_{n-p+1}}
\end{aligned}
$$

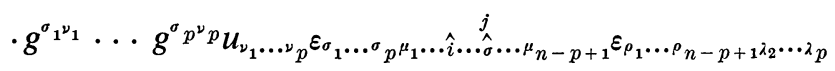

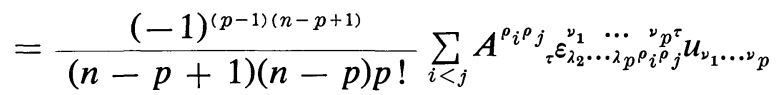

$$
\begin{aligned}
& =-\nabla^{\tau} \varphi^{\rho}{ }_{\tau} u_{\rho \lambda_{2} \ldots \lambda_{p}}-\sum_{\alpha=2}^{p}(-1)^{\alpha} \nabla^{\rho} \varphi_{\lambda_{\alpha}}^{\sigma} u_{\rho \sigma \lambda_{2} \ldots \hat{\alpha} \ldots \lambda_{p}} \\
& =-(i(\delta \varphi) u-\vartheta u)_{\lambda_{2} \cdots \lambda_{p}} \text {. }
\end{aligned}
$$

Theorem 2.6. In an almost Hermitian space, we have the following relations:

$$
\begin{aligned}
& \boldsymbol{\partial}=(d-\sqrt{-1} \Gamma) / 2, \\
& \mathfrak{D}=(\delta-\sqrt{-1} D) / 2 .
\end{aligned}
$$

Proof. Using

$$
\Pi_{\lambda}{ }^{\rho} \Pi_{\rho}{ }^{\mu}=\Pi_{\lambda}{ }^{\mu}, \quad \Pi_{\lambda}{ }^{\rho} \bar{\Pi}_{\rho}{ }^{\mu}=0, \quad \bar{\Pi}_{\lambda}{ }^{\rho} \Pi_{\rho}{ }^{\mu}=0, \quad \bar{\Pi}_{\lambda}{ }^{\rho} \bar{\Pi}_{\rho}{ }^{\mu}=\bar{\Pi}_{\lambda}{ }^{\mu},
$$

we have 


$$
\begin{aligned}
& (\partial u)_{\lambda_{0} \cdots \lambda_{p}}
\end{aligned}
$$

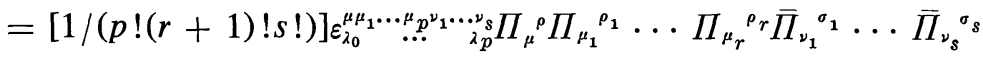

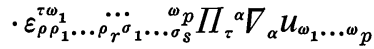

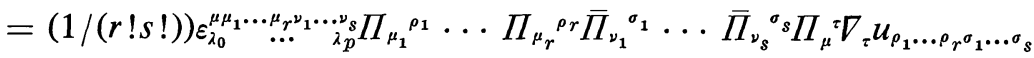

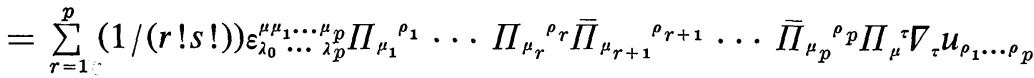

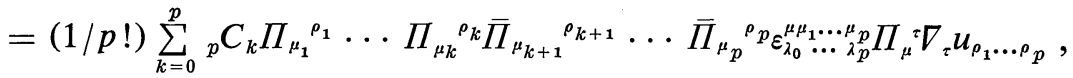

which is reduced to, in consequence of (1.11),

$$
\begin{aligned}
& (\partial u)_{\lambda_{0} \ldots \lambda_{p}}=(1 / p !) \varepsilon_{\lambda_{0} \ldots \lambda_{p} \rho_{1} \ldots \rho}^{\mu \rho}\left(\Pi_{\mu}{ }^{\tau} \nabla_{\tau} u_{\rho_{1} \ldots \rho_{p}}\right)
\end{aligned}
$$

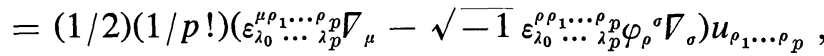

giving (2.6). As for (2.7), from the definition we have

$$
\begin{aligned}
& (\mathfrak{D} u)_{\lambda_{2} \cdots \lambda_{p}}
\end{aligned}
$$

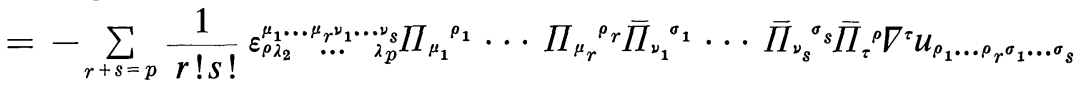

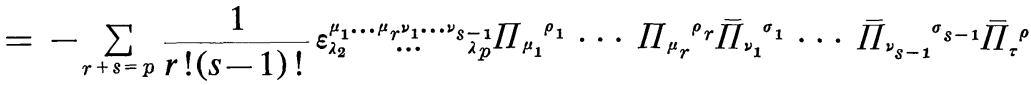

$$
\begin{aligned}
& \cdot \nabla^{\tau} u_{\rho \rho_{1} \ldots \rho_{r} \sigma_{1} \ldots \sigma_{s-1}} \\
& =-\frac{1}{(p-1) !} \sum_{r=1}^{p-1} \frac{(p-1) !}{r !(p-r-1) !} \varepsilon_{\lambda_{2}}^{\mu_{1} \ldots{ }^{\mu}{ }^{\mu}{ }^{p}-1} \Pi_{p} \Pi_{\mu_{1}}{ }^{\rho_{1}} \ldots \Pi_{\mu_{r}}{ }^{\rho} r \\
& \cdot \bar{\Pi}_{\mu_{r+1}}{ }^{\rho_{r+1}} \ldots \bar{\Pi}_{\mu_{p-1}{ }^{\rho_{p-1}} \bar{\Pi}_{\tau}{ }^{\rho} \nabla^{\tau} u_{\rho \rho_{1} \ldots \rho} \ldots \rho-1},
\end{aligned}
$$

which becomes, due to (1.11),

$$
\begin{aligned}
(\mathfrak{D} u)_{\lambda_{2} \cdots \lambda_{p} p} & =(-1 /(p-1) !) \varepsilon_{\lambda_{2}{ }^{\rho} \cdots \rho^{p} p \bar{\lambda}_{p}^{1}}\left(\bar{\Pi}_{\tau}^{\rho} \nabla^{\tau} u_{\rho \rho_{1} \cdots \rho_{p-1}}\right) \\
& =-\bar{\Pi}_{\tau}{ }^{\rho} \nabla^{\tau} u_{\rho \lambda_{2} \cdots \lambda_{p}}=(-1 / 2)\left(\nabla^{\rho}+\sqrt{-1} \varphi_{\tau}{ }^{\rho} \nabla^{\tau}\right) u_{\rho \lambda_{2} \cdots \lambda_{p}},
\end{aligned}
$$

giving (2.7).

Corollary 2.6. In an almost Hermitian space, we have

$$
\mathfrak{D}=-* \partial * \text {. }
$$

Proof. Since the dimension $n$ of the space is even, from (1.3) we have $\delta=-* d *$ for any $p$-form. Hence (2.8) is an immediate consequence of Theorem 2.6 and Lemma 2.1.

Corollary 2.7. In an almost Hermitian space, we have

$$
\begin{aligned}
& \overline{\boldsymbol{\partial}}=(d+\sqrt{-1} \Gamma) / 2, \\
& \overline{\mathfrak{D}}=(\delta+\sqrt{-1} D) / 2 .
\end{aligned}
$$


Next we study the complex operators $\partial$ and $\mathscr{D}$. Since $\partial$ is a skew-derivation, we first show how $\partial$ is expressed on $\mathscr{F}^{0}$ and $\mathscr{F}^{1}$. Let $f$ be a 0 -form. Then we have

$$
(\partial f)_{\lambda}=\prod_{1,0}{ }^{\rho} \nabla_{\rho} f=(1 / 2)(d f-\sqrt{-1} \Gamma f)=(\partial f)_{\lambda},
$$

and therefore

$$
\partial f=\partial f=(d f-\sqrt{-1} \Gamma f) / 2 .
$$

Let $u$ be a 1 -form. Then we shall show that the relation

$$
\partial u=(1 / 2)(d u-\sqrt{-1}(\Gamma-\gamma) u)
$$

is satisfied. For this purpose, we need the following formulas:

$$
\begin{aligned}
& \prod_{2,0} w=(1 / 4)(w-\Psi w-\sqrt{-1} \Phi w) \\
& \prod_{1,1} w=(1 / 2)(w+\Psi w) \\
& \prod_{0,2} w=(1 / 4)(w-\Psi w+\sqrt{-1} \Phi w)
\end{aligned}
$$

where $w$ is any 2-form. By decomposing the 1-form $u$ as $u=u_{1}+u_{2}$, where $u_{1}$ (resp. $u_{2}$ ) denotes the part of type $(1,0)$ (resp. type $\left.(0,1)\right)$, we have

$$
\begin{aligned}
& d_{1} u_{1}=\prod_{2,0} d u_{1}=(1 / 4)\left(d u_{1}-\Psi d u_{1}-\sqrt{-1}\left(\Phi d u_{1}\right)\right) \\
& d_{2} u_{1}=0 \\
& \bar{d}_{2} u_{1}=\prod_{0,2} d u_{1}=(1 / 4)\left(d u_{1}-\Psi d u_{1}+\sqrt{-1}\left(\Phi d u_{1}\right)\right), \\
& d_{1} u_{2}=\prod_{1,1} d u_{2}=(1 / 2)\left(d u_{2}+\Psi\left(d u_{2}\right)\right), \\
& d_{2} u_{2}=\prod_{2,0} d u_{2}=(1 / 4)\left(d u_{2}-\Psi d u_{2}-\sqrt{-1}\left(\Phi d u_{2}\right)\right) \\
& \bar{d}_{2} u_{2}=0 .
\end{aligned}
$$

and therefore, by virtue of (1.16),

which imply

$$
\begin{aligned}
& \partial u_{1}=-\sqrt{-1}\left(\Phi d u_{1}\right) / 2, \\
& \partial u_{2}=d u_{2}-\sqrt{-1}\left(\Phi d u_{2}\right) / 2,
\end{aligned}
$$

$$
\begin{aligned}
\partial u & =d \prod_{0,1} u-\sqrt{-1}(\Phi d u) / 2 \\
& =(1 / 2)(d u+\sqrt{-1}(d \Phi u-\Phi d u)) .
\end{aligned}
$$

Making use of (2.3), we hence arrive at (2.12). 
Now we consider an operator

$$
\partial^{\prime}=(1 / 2)(d-\sqrt{-1}(\Gamma-\gamma))
$$

from $\mathscr{F}_{c}{ }^{p}$ to $\mathscr{F}_{c}{ }^{p+1}$. Since $\gamma f$ vanishes for any 0-form $f,(2.11)$ and (2.12) show that $\partial$ and $\partial^{\prime}$ coincide on any form of degrees 0 and 1 . Owing to Lemma 2.1 and Corollary 2.3, the operator $\partial^{\prime}$ is a skew-derivation. Therefore $\partial$ coincides with $\partial^{\prime}$ on all forms of any degree. By applying the adjoint operator $*$ and using Lemmas 2.1 and 2.5, we obtain

$$
-* \partial *=(1 / 2)(\delta-\sqrt{-1}(D-\vartheta-i(\delta \varphi))) .
$$

Since $\mathfrak{D}$ is defined by $-* \partial *$, we have proved

Theorem 2.8. In an almost Hermitian space, we have

$$
\begin{aligned}
& \partial=(1 / 2)(d-\sqrt{-1}(\Gamma-\gamma)), \\
& \mathfrak{D}=(1 / 2)(\delta-\sqrt{-1}(D-\vartheta-i(\delta \varphi)) .
\end{aligned}
$$

Remark. As is easily seen, $\partial$ and $\partial$ coincide if and only if the operator $\gamma$ vanishes. This condition is equivalent to

$$
\nabla_{\lambda} \varphi_{\mu \nu}=\nabla_{\mu} \varphi_{\lambda \nu}
$$

As the tensor $\nabla_{\lambda} \varphi_{\mu \nu}$ is skew-symmetric in the indices $\mu, \nu$, from (2.16) we can easily see that $\nabla_{\lambda} \varphi_{\mu \nu}$ vanishes, and therefore that the structure is Kaehlerian. Hence the operator $\partial$ coincides with $\partial$ if and only if the structure is Kaehlerian.

\section{Theorems}

In [2], the integrability condition of the almost complex structure is defined by $\partial^{2}=0$. Since, from Theorem 2.8 ,

$$
\partial^{2}=(1 / 4)\left(-(\Gamma-\gamma)^{2}+\sqrt{-1}(d \Gamma+\Gamma d-d \gamma-\gamma d)\right),
$$

of which the imaginary part vanishes because of Corollary 2.4 , we obtain

$$
\partial^{2}=(-1 / 4)(\Gamma-\gamma)^{2},
$$

which is a real operator. Hence we reach

Theorem 3.1. In an almost Hermitian space, in order that the structure be integrable it is necessary and sufficient that

$$
(\Gamma-\gamma)^{2}=0 \text {. }
$$

We shall use the operator $\Gamma$ to characterize a Kaehlerian structure by showing that an almost Hermitian structure is Kaehlerian if and only if the operator 
$\Gamma^{2}$ vanishes. As $\Gamma$ is a skew-derivation, $\Gamma^{2}$ is a derivation. Therefore if $\Gamma^{2}$ vanishes for all forms of degrees 0 and 1 , then it vanishes for all forms of any degree. If we take a 0 -form $f$ and a 1 -form $u=\left(u_{\lambda}\right)$, then we have

$$
\begin{aligned}
\left(\Gamma^{2} f\right)_{\lambda \mu} & =\left(\varphi_{\lambda}{ }^{\rho} \nabla_{\rho} \varphi_{\mu}{ }^{\sigma}-\varphi_{\mu}{ }^{\rho} \nabla_{\rho} \varphi_{\lambda}{ }^{o}\right) \nabla_{\sigma} f \\
\left(\Gamma^{2} u\right)_{\lambda \mu \nu} & =\underset{\lambda, \mu, \nu}{\subseteq}\left(\varphi_{\lambda}{ }^{\rho} \nabla_{\rho} \varphi_{\mu}{ }^{\sigma}-\varphi_{\mu}{ }^{\rho} \nabla_{\rho} \varphi_{\lambda}{ }^{\sigma}\right) V_{\sigma} u_{\nu}+\underset{\lambda, \mu, \nu}{\subseteq}\left(\varphi_{\lambda}{ }^{\rho} \varphi_{\mu}{ }^{\sigma} R_{\rho \sigma \nu}{ }^{\tau}\right) u_{\tau},
\end{aligned}
$$

where $\underset{\nu, \mu, \nu}{\mathfrak{S}}$ indicates that the terms are summed cyclicly with respect to $\lambda, \mu, \nu$. Thus the condition $\Gamma^{2}=0$ is equivalent to the relations

$$
\begin{aligned}
\varphi_{\lambda}{ }^{\rho} \nabla_{\rho} \varphi_{\mu}{ }^{\sigma}-\varphi_{\mu}{ }^{\rho} \nabla_{\rho} \varphi_{\lambda}{ }^{\sigma} & =0, \\
\underset{\lambda, \mu, \nu}{S} \varphi_{\lambda}{ }^{\rho} \varphi_{\mu}{ }^{\sigma} R_{\rho \sigma \nu}{ }{ }^{\sigma} & =0 .
\end{aligned}
$$

Theorem 3.2. In a Kaehlerian space, the operator $\Gamma^{2}$ always vanishes.

Proof. Since the complex structure $\varphi_{\lambda}{ }^{\mu}$ is a covariant constant in a Kaehlerian space, (3.1) follows obviously. Moreover we have (see, for instance, $[1,(4.13)])$

$$
\varphi_{\lambda}{ }^{\rho} R_{\rho \mu \nu}{ }^{\omega}=\varphi_{\mu}{ }^{\rho} R_{\rho \lambda \nu}{ }^{\omega},
$$

and therefore

$$
\varphi_{\lambda}{ }^{\rho} \varphi_{\mu}{ }^{\sigma} R_{\rho \sigma \nu}{ }^{\omega}=R_{\lambda \mu \nu}{ }^{\omega}
$$

which easily gives (3.2).

In order to show the validity of the converse of Theorem 3.2 we need the following lemmas. The almost Hermitian structure is said to be almost semiKaehlerian if the fundamental 2-form is co-closed, that is, if the relation $\nabla^{\lambda} \varphi_{\lambda \mu}=0$ holds.

Lemma 3.3. If $\Gamma^{2}=0$ in an almost Hermitian space, then the structure is almost semi-Kaehlerian.

Proof. Transvecting (3.1) with $\varphi_{\tau}^{2}$, we have

$$
\nabla_{\tau} \varphi_{\mu}{ }^{\sigma}+\varphi_{\tau}{ }^{2} \varphi_{\mu}{ }^{\rho} \nabla_{\rho} \varphi_{\lambda}{ }^{\sigma}=0
$$

Contracting $\tau$ and $\sigma$ and noting $\varphi^{\lambda \sigma} \nabla_{\rho} \varphi_{\lambda \sigma}=0$, we thus prove our lemma.

Lemma 3.4. If $\Gamma^{2}=0$ in an almost Hermitian space, then we have

$$
\begin{aligned}
\mathfrak{S}{ }_{\lambda, \mu, \nu}{ }_{i}{ }^{\rho} R_{\mu \nu \rho}{ }^{\omega} & =0, \\
(1 / 2) \varphi^{\rho \sigma} R_{\rho \sigma \lambda}{ }^{\mu}+\varphi_{\lambda}{ }^{\rho} R_{\rho}{ }^{\mu} & =0, \\
\varphi_{\lambda}{ }^{\rho} R_{\rho \mu}+\varphi_{\mu}{ }^{\rho} R_{\rho \lambda} & =0 .
\end{aligned}
$$


Proof. From (3.2), we get

$$
\varphi_{\lambda}{ }^{\rho} \varphi_{\mu}{ }^{\sigma} \varphi_{\nu}{ }^{\tau} R_{\rho \sigma \tau}{ }^{\omega}=\varphi_{\lambda}{ }^{\rho} R_{\nu \rho \mu}{ }^{\omega}-\varphi_{\mu}{ }^{\rho} R_{\nu \rho \lambda}{ }^{\omega} .
$$

Taking the sum of the terms of (3.6) cyclicly with respect to the indices $\lambda, \mu, \nu$, we have

$$
0=\underset{\lambda, \mu, \nu}{\mathbb{S}} \varphi_{\lambda}{ }^{\rho} \varphi_{\mu}{ }^{\sigma} \varphi_{\nu}{ }^{\tau} R_{\rho \sigma \tau}{ }^{\omega}=\underset{\lambda, \mu, \nu}{\mathbb{S}} \varphi_{\lambda}{ }^{\rho} R_{\mu \nu \rho}{ }^{\omega}
$$

giving (3.3). Contraction of $\lambda$ and $\omega$ in (3.3) yields

$$
\varphi^{\lambda \rho} R_{\lambda \rho \mu \nu}+\varphi_{\mu}{ }^{\rho} R_{\rho \nu}-\varphi_{\nu}{ }^{\rho} R_{\rho \mu}=0 .
$$

On the other hand, from (3.6) we get

$$
-\varphi_{\nu}{ }^{\rho} \varphi_{\lambda}{ }^{\sigma} \varphi_{\mu}{ }^{\tau} R_{\rho \sigma \tau}{ }^{\omega}=\varphi_{\lambda}{ }^{\rho} R_{\nu \rho \mu}{ }^{\omega}-\varphi_{\nu}{ }^{\rho} R_{\lambda \rho \mu}{ }^{\omega}-\varphi_{\mu}{ }^{\rho} R_{\nu \lambda \rho}{ }^{\omega},
$$

which can easily be reduced to (3.4) by contracting with $g^{\lambda \mu}$. Finally, the relation (3.5) follows readily from (3.7) and (3.4).

Lemma 3.5. If $\Gamma^{2}=0$ in an almost Hermitian space, then

$$
\nabla^{\lambda} \varphi^{\mu \nu} \nabla_{\nu} \varphi_{\lambda \omega}=0
$$

Proof. Since the structure is co-closed by Lemma 3.3, differentiating (3.1) by $\nabla_{\lambda}$, we have

$$
\varphi^{\lambda \rho} \nabla_{\lambda} \nabla_{\rho} \varphi_{\mu}{ }^{\sigma}=\nabla^{\lambda} \varphi_{\mu}{ }^{\rho} \nabla_{\rho} \varphi_{\lambda}{ }^{\sigma}+\varphi_{\mu}{ }^{\rho}\left(R_{\lambda \rho \varepsilon}{ }^{\lambda} \varphi^{\varepsilon \sigma}+R_{\lambda \rho \varepsilon}{ }^{\sigma} \varphi^{\lambda \varepsilon}\right) .
$$

Owing to (3.4) and (3.5) the left hand side becomes

$$
\begin{aligned}
(1 / 2) \varphi^{\lambda \rho}\left(\nabla_{\lambda} \nabla_{\rho} \varphi_{\mu}{ }^{\sigma}-\nabla_{\rho} \nabla_{\lambda} \varphi_{\mu}{ }^{\sigma}\right) & =(-1 / 2) \varphi^{\lambda \rho} R_{\lambda \rho \mu}{ }^{\sigma} \varphi_{\varepsilon}{ }^{\sigma}+(1 / 2) \varphi^{\lambda \rho} R_{\lambda \rho \varepsilon}{ }^{\sigma} \varphi_{\mu}{ }^{\sigma} \\
& =-R^{\sigma}{ }_{\mu}+R^{\sigma}{ }_{\mu}=0 .
\end{aligned}
$$

In the same way, the second and third terms on the right hand side are reduced to $-R^{\sigma}{ }_{\mu}$ and $R_{\mu}{ }^{\sigma}$, respectively and thus we have (3.8).

Theorem 3.6. In an almost Hermitian space, if the operator $\Gamma^{2}$ vanishes everywhere, then the space is Kaehlerian.

Proof. At first we show that

$$
\varphi^{\mu \nu} \nabla^{\rho} \nabla_{\rho} \varphi_{\mu \nu}=0 .
$$

In fact, by virtue of (3.1) we have

$$
\nabla_{\lambda} \varphi_{\mu}{ }^{\sigma}=\varphi_{\mu}{ }^{\rho} \varphi_{\tau}{ }^{\rho} \nabla_{\rho} \varphi_{\lambda}{ }^{\tau},
$$

which and (3.8), (3.5) imply

$$
\nabla^{\lambda} \nabla_{\lambda} \varphi_{\mu}{ }^{\sigma}=\varphi_{\mu}{ }^{\rho} \nabla^{\lambda} \varphi_{\tau}{ }^{\rho} \nabla_{\rho} \varphi_{\lambda}{ }^{\tau} .
$$


contracting with $\varphi^{\mu}{ }_{\sigma}$ and applying Lemma 3.5 we hence obtain (3.9).

From (3.9) follows immediately

$$
\nabla^{\lambda} \varphi^{\mu \nu} \nabla_{\lambda} \varphi_{\mu \nu}=(1 / 2) \nabla^{\lambda} \nabla_{\lambda}\left(\varphi^{\mu \nu} \varphi_{\mu \nu}\right)-\varphi^{\mu \nu} \nabla^{\lambda} \nabla_{\lambda} \varphi_{\mu \nu}=0,
$$

which means $\nabla_{\lambda} \varphi_{\mu \nu}=0$, showing the structure to be Kaehlerian.

Now we define the complex Laplace-Beltrami operators $\square$ and $\square$ by

$$
\begin{aligned}
& \square=\mathfrak{D} \bar{\partial}+\bar{\partial} \mathfrak{D}, \\
& \square=\mathfrak{D} \bar{\partial}+\bar{\partial} \mathfrak{D} .
\end{aligned}
$$

Then using Theorems 2.6 and 2.8, we can calculate the real and imaginary parts of the operators $\square$ and $\square$. First, for the operator $\square$ we have

Theorem 3.8. If the operator $\square$ in an almost Hermitian space is real on all forms of degrees 0 and 1, then the space is Kaehlerian (cf. [1]).

Proof. From Theorem 2.6 and Corollary 2.7 it follows that

$$
4 \square=\Delta+(D \Gamma+\Gamma D)+\sqrt{-1}(\delta \Gamma+\Gamma \delta-d D-D d) .
$$

Therefore by the assumption we have, on $\mathscr{F}^{0}$ and $\mathscr{F}^{1}$,

$$
\delta \Gamma+\Gamma \delta-d D-D d=0,
$$

which implies, for a 0 -form $f, \delta \Gamma f-D d f=\delta \Gamma f=0$, since $\delta f=D f=0$. Thus $\nabla^{\rho} \varphi_{\rho}{ }^{\sigma} \nabla_{\sigma} f=0$ for all 0 -form $f$, due to $\nabla_{\rho} \nabla_{\sigma} f=\nabla_{\sigma} \nabla_{\rho} f$. By choosing orthogonal geodesic local coordinates $x^{1}, \ldots, x^{n}$ at a point $P$ and putting $f=x^{\sigma}$, we can easily see that $\nabla^{\rho} \varphi_{\rho}{ }^{\sigma}=0$ at every point $P$, which means that the space is almost semi-Kaehlerian. Application of (3.10) to a 1-form $u=\left(u_{2}\right)$ then gives

$$
-\nabla^{\rho} \varphi_{\rho}{ }^{\sigma} \nabla_{\sigma} u_{\lambda}+\left(\nabla^{\sigma} \varphi_{\lambda}{ }^{\rho}+\nabla_{\lambda} \varphi^{\sigma \rho}\right) \nabla_{\rho} u_{\sigma}+\left(\frac{1}{2} \varphi^{\rho \sigma} R_{\rho \sigma \lambda}{ }^{\tau}+\varphi_{\lambda}{ }^{\rho} R_{\rho}{ }^{\tau}\right) u_{\tau}=0,
$$

where the first term is zero. Now to the above equation we first apply $u=$ $\delta_{\mu}^{\lambda} d x^{\mu}$ for a fixed $\lambda$, for which $\left(\nabla_{\rho} u_{\sigma}\right)_{p}=0$, and then apply $u=x^{\rho} d x^{\mu}$ for any fixed distinct $\rho$ and $\mu$, for which $\left(\nabla_{\sigma} u_{\tau}\right)_{p}=\delta_{\sigma}^{\rho} \delta_{\tau}^{\mu}$.

$$
\begin{gathered}
\nabla_{\lambda} \varphi_{\mu}{ }^{\sigma}+\nabla_{\mu} \varphi_{\lambda}{ }^{\sigma}=0, \\
(1 / 2) \varphi^{\rho \sigma} R_{\rho \sigma \lambda \mu}+\varphi_{\lambda}{ }^{\rho} R_{\rho \mu}=0 .
\end{gathered}
$$

(3.11) shows that the space is almost Tachibana. From Kotô's theorem (see K. Yano [5, p. 180, Theorem 2.5]) if (3.12) is satisfied in an almost Tachibana space, then the space is Kaehlerian. Thus Theorem 3.8 is proved.

Next we consider the operator $\square$. From Theorem 2.8, we have

$$
\begin{gathered}
4 \square=\Delta+(\Gamma-\gamma)(D-\vartheta-i(\delta \varphi))+(D-\vartheta-i(\delta \varphi))(\Gamma-\gamma) \\
-\sqrt{-1}[(D-\vartheta-i(\delta \varphi)) d+d(D-\vartheta-i(\delta \varphi)) \\
-\delta(\Gamma-\gamma)-(\Gamma-\gamma) \delta] .
\end{gathered}
$$


Theorem 3.9. If the complex Laplace-Beltrami operator $\square$ for an almost Hermitian structure is real on all forms of degree 0 , then the structure is almost semi-Kaehler.

Proof. For any 0-form $f$, since $\delta f=D f=0$ and $\vartheta f=\gamma f=0$, by our assumption from (3.13) follows immediately

$$
(D-\vartheta-i(\delta \varphi)) d f-\delta \Gamma f=0,
$$

which further reduces to, because of $D d f=0$ and $\vartheta d f=0$,

$$
i(\delta \varphi) d f-\delta \Gamma f=0,
$$

implying that $\nabla^{\rho} \varphi_{\rho}{ }^{\sigma} \nabla_{\sigma} f=0$ for any 0 -form $f$. Thus $\nabla^{\rho} \varphi_{\rho}{ }^{\sigma}=0$, and the theorem is proved.

Theorem 3.10. If the operator $\square$ for an almost Hermitian structure is real on all forms of degrees 0 and 1 , then the structure is Kaehlerian.

Proof. By definition, for a 1-form $u$ we have $\vartheta u=0$, and

$$
\begin{aligned}
(\vartheta d u)_{\lambda} & =\nabla^{\rho} \varphi_{\lambda}^{\sigma}{ }_{\lambda} \nabla_{\rho} u_{\sigma}-\nabla^{\rho} \varphi^{\sigma}{ }_{\lambda} \nabla_{\sigma} u_{\rho}, \\
(\delta \gamma u)_{\lambda} & =\nabla^{\rho} \nabla_{\lambda} \varphi_{\rho}{ }^{\sigma} u_{\sigma}-\nabla^{\rho} \nabla_{\rho} \varphi_{\lambda}{ }^{\sigma} u_{\sigma}+\nabla_{\lambda} \varphi_{\rho}{ }^{\sigma} \nabla^{\rho} u_{\sigma}-\nabla_{\rho} \varphi_{\lambda}{ }^{\sigma} \nabla^{\rho} u_{\sigma} .
\end{aligned}
$$

As the structure is almost semi-Kaehlerian due to Theorem 3.9, from the proof of Theorem 3.8 we obtain

$$
2\left(\nabla_{\lambda} \varphi^{\rho \sigma}-\nabla^{\sigma} \varphi_{\lambda}{ }^{\rho}\right) \nabla_{\rho} u_{\sigma}+\left(-\nabla^{\rho} \nabla_{\rho} \varphi_{\lambda}{ }^{\tau}-\varphi_{\lambda}{ }^{\rho} R_{\rho}{ }^{\tau}+R_{\lambda}{ }^{\rho} \varphi_{\rho}{ }^{\tau}\right) u_{\tau}=0,
$$

which implies

$$
\nabla_{\lambda} \varphi_{\mu}{ }^{\rho}+\nabla_{\mu} \varphi_{\lambda}{ }^{\rho}=0, \quad \nabla^{\rho} \nabla_{\rho} \varphi_{\lambda}{ }^{\tau}+\varphi_{\lambda}{ }^{\rho} R_{\rho}{ }^{\tau}-R_{\lambda}{ }^{\rho} \varphi_{\rho}{ }^{\tau}=0,
$$

by an argument similar to that in the proof of Theorem 3.9. Contracting with $\varphi_{\tau}^{\lambda}$ in the last equation gives (3.9) and therefore $\nabla_{\lambda} \varphi_{\mu_{\nu}}=0$ in the same way as in the proof of Theorem 3.6. Hence Theorem 3.10 is proved.

\section{Bibliography}

[1] C. C. Hsiung, Structures and operators on almost-Hermitian manifolds, Trans. Amer. Math. Soc. 122 (1966) 136-152.

[2] K. Kodaira \& D. C. Spencer, On the variation of almost-complex structure, A Sympos. in Honor of S. Lefschetz, Princeton University Press, Princeton 1957, $139-150$.

[3] A. Lichnerowicz, Théorie globale des connexions et des groupes d'holonomie, Cremonese, Rome, 1955.

[4] Y. Ogawa, On C-harmonic forms in a compact Sasakian space, Tôhoku Math. J. 19 (1967) 267-296.

[ 5 ] K. Yano, Differential geometry on complex and almost complex spaces, Pergamon, New York, 1965. 
